\title{
Topological Configurations of Optical Phase Singularities
}

\author{
M. R. Dennis \\ School of Mathematics, University of Southampton, Highfield, Southampton SO17 1BJ, \\ $U K$ \\ mark.dennis@physics.org
}

\begin{abstract}
Geometric and topological properties of phase singularity lines in threedimensional complex scalar wavefields are discussed. In particular, their role as the intersections of the zero contour surfaces of the real and imaginary parts of the field gives numerous insights into 3D vortex topology. In addition, complex scalar wavefields (i.e. solutions of the three-dimensional Helmholtz and paraxial equations) are compared to more general complex scalar fields, including those arising naturally from algebraic geometry.
\end{abstract}

\section{Introduction}

Controlling and manipulating the configuration of phase singularity lines in a threedimensional optical field is one of the most challenging problems in holographic beam shaping. Phase singularities are also known as nodal lines, wave dislocations and optical vortices, and are deeply connected with the underlying geometry and topology of the field 1, 2, 3, 4. In particular, as systems of curved lines in three dimensions, oriented by their vorticity (topological current), a range of topological configurations are possible in principle, such as infinite lines, closed loops, knots, links, braids etc. 1, 5, 6, 7, 8, 9, 10. In linear optical fields, the vortex lines arise purely by interference. Despite the linearity of the wave superposition, the details of the vortex configuration - and their response to any change in the superposed waves (such as amplitude, phase or propagation direction) - is rather nonlinear, and therefore hard to control. This contrasts with the holographic manipulation of bright regions (e.g. Ref. 11), which merely requires superposing additional waves in phase with the high intensity regions.

Mathematically, the problem of controlling optical vortex topology corresponds to controlling the nodal lines in a three-dimensional complex scalar field, which satisfies the time-independent scalar wave equation (Helmholtz equation) or the paraxial equation, as polarized components of the electric field in the appropriate regime of Maxwell's equations for optical fields. Such complex waves $\psi=\psi(\boldsymbol{r})$ may be be considered as the complex sum of two real wave fields,

$$
\psi=\xi+\mathrm{i} \eta,
$$

and the nodal lines - places $\boldsymbol{r}$ where $\psi(\boldsymbol{r})=0$ - occur at the intersections of the nodal surfaces (wavefronts) of the real fields $\xi(\boldsymbol{r}), \eta(\boldsymbol{r})$. Thus unusual line topologies of vortex lines require particular topologies of the surfaces $\xi=0, \eta=0$, and vice versa. For instance, a two-component link (Hopf link) of vortex loops can only arise in the intersection of wave surfaces of nonzero genus, such as a torus (as considered in Ref. [12]) or a noncompact torus [8].

\footnotetext{
${ }^{1}$ Current Address: H. H. Wills Physics Laboratory, Tyndall Avenue, Bristol BS8 1TL, UK
} 


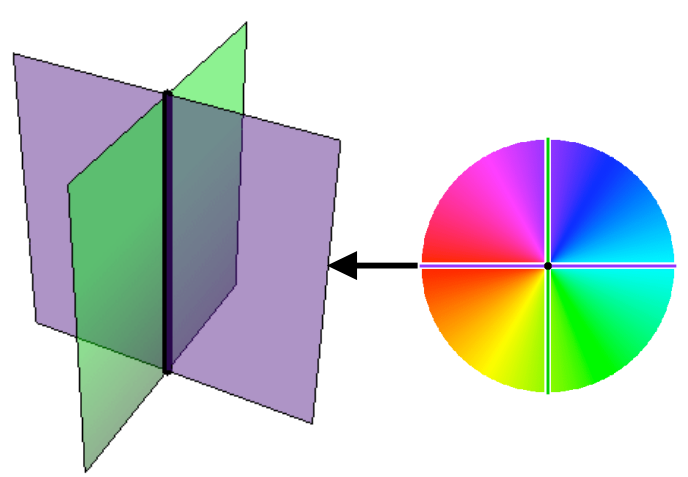

Fig. 1 An infinite, straight vortex as the intersection of two flat planes, the real and imaginary zero contours of $x+\mathrm{i} y$. The colour scheme implicit here, and used explicitly in the following figures, is the green surface represents $\xi=0$, the blue surface $\eta=0$. A section of the field transversely through the vortex cyclically goes through all phases, represented as the $2 \pi$ periodic hues on the colour wheel.

Here, I will consider the mathematics of complex scalar fields containing different topological configurations of nodal lines. Although most of the fields considered here are too algebraically simple to satisfy the wave equation, their topology gives insights into what might be possible in complex wave fields, and possible relationships will be discussed when appropriate.

The simplest example of two intersecting surface is provided by the field $\psi=$ $x+\mathrm{i} y$. This is a local solution to laser modes with an on-axis strength one vortex (e.g. Laguerre-Gauss $\mathrm{LG}_{10}$ modes and $J_{1}$ Bessel beams), and is the intersection of two planes (the $y z$-and $x z$-planes) on the $z$-axis (Fig. 1). The vortex strength is the total phase accumulated in units of $2 \pi$ in an oriented loop around the vortex; the orientation of the surfaces (i.e. the direction of the normal, pointing to the positive side) is required to determine the direction. This is conveniently given by the vorticity $\boldsymbol{\Omega}$,

$$
\boldsymbol{\Omega}=\nabla \xi \times \nabla \eta=\frac{1}{2} \nabla \times \boldsymbol{j},
$$

where $\boldsymbol{j}=\operatorname{Im} \psi^{*} \nabla \psi$ is the current flow associated with the field. In terms of the zero contour surfaces, $\boldsymbol{\Omega}$ must lie in the direction of the vortex line from the first equality, as it is perpendicular to both $\nabla \xi$ and $\nabla \eta$, so lies in the intersection of the two surfaces.

Vortices of higher strength are also possible simply by taking a positive integer power $(x+\mathrm{i} y)^{\ell}$, as with Laguerre-Gauss and Bessel beams of azimuthal order greater than one. In this case, the sequence of phases around the vortex line repeats $\ell$ times, and the real and imaginary contour surfaces intersect themselves as well as each other along the vortex (as shown, for example, in Fig. 2). Along such highorder vortex lines, the vorticity $\boldsymbol{\Omega}$ vanishes, and in general, a field containing such a strength $\ell$ singularity must locally satisfy $\ell(\ell+1)$ conditions [4, 5]; as with Bessel and Laguerre-Gauss beams, they usually occur in optics as azimuthal modes of beam solutions which have been separated in cylindrical polar coordinates.

Another feature of nodal lines that is not necessarily revealed in the intersection of zero contour surfaces is the anisotropy of the vortex core [4, 8, 13, 14, 15]; the phase lines in the neighbourhood of a vortex are usually squeezed, in a way related to the anisotropy of the intensity growing away from the node [15] (Fig. 3b). For unit strength nodes, there is always a local phase (gauge) transformation $\psi \rightarrow \psi \exp (\mathrm{i} \alpha)$ 


\section{Topologica}

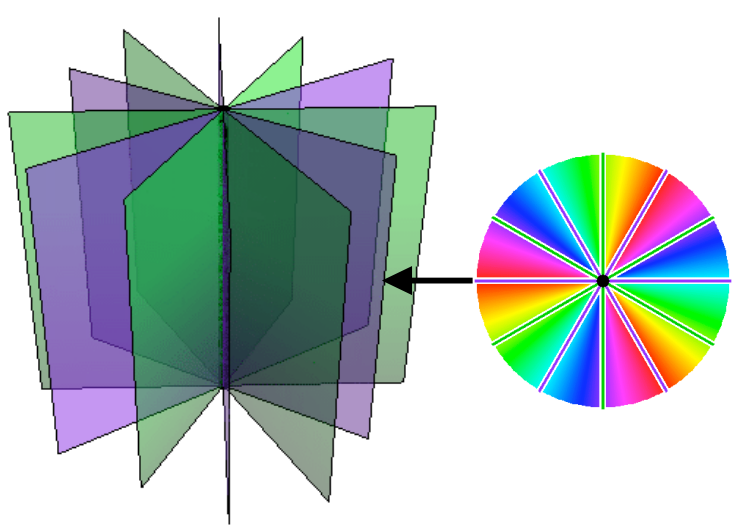

Fig. 2 The threefold self-intersecting phase surfaces of a strength 3 phase singularity $(x+\mathrm{i} y)^{3}$. A transverse section goes through all phases three times, i.e. $6 \pi$.

which will make the real and imaginary zero contours perpendicular [4]; the lengths of the gradients $\nabla \xi$ and $\nabla \eta$ then determine the anisotropy. In the plane transverse to the vortex line - taken to be the $x y$-plane - any unit-strength vortex (with $\boldsymbol{\Omega} \cdot \hat{\boldsymbol{z}}>0)$ can be written as a sum $a(x+\mathrm{i} y)+b(x-\mathrm{i} y)$ with $|a|>|b|$ (the complex ratio $a / b$ stereographically projects to the Poincaré-like sphere describing the vortex anisotropy [4, 8]). In fact, local analysis shows that in any solution of the Helmholtz equation, or the paraxial equation (provided the vortex line is not perpendicular to $\hat{\boldsymbol{z}}$ ), any high-strength vortex of strength $\ell$ must also be of similar form, $a(x+\mathrm{i} y)^{\ell}+b(x-\mathrm{i} y)^{\ell}$, rather than a more general polynomial in $x$ and $y[5]$. The intensity and phase in the neighbourhood of a strength $\ell$ wave dislocation are therefore $\ell$-fold symmetric (Fig. 3p), the precise dependence on radius being given by the appropriate generalized ellipse [4.

Of course, the total nodal line configuration of a product of complex scalar fields is the union of the nodal lines in the individual fields being multiplied. Although the topology of the nodal lines themselves is not affected by the multiplication, the topology of the real and imaginary zero surfaces is. A familiar example of the effect of a multiplying field on the example field $x+\mathrm{i} y$ is the so-called screw

a

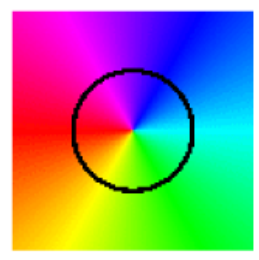

b

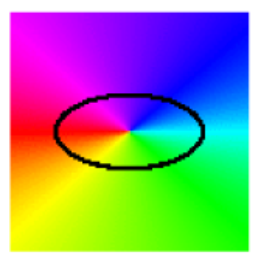

C

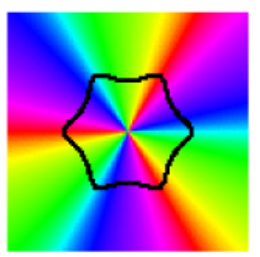

Fig. 3 The phase pattern (hue plot) and low-lying intensity contours (black line) for three phase singularities in the plane transverse to $\boldsymbol{\Omega}$. (a) Isotropic unit strength dislocation $x+\mathrm{i} y$; (b) anisotropic dislocation $x+2 \mathrm{i} y=3 / 2(x+\mathrm{i} y)-1 / 2(x-\mathrm{i} y)$; (c) anisotropic strength 3 dislocation $3 / 2(x+\mathrm{i} y)^{3}-1 / 2(x-\mathrm{i} y)^{3}$. 


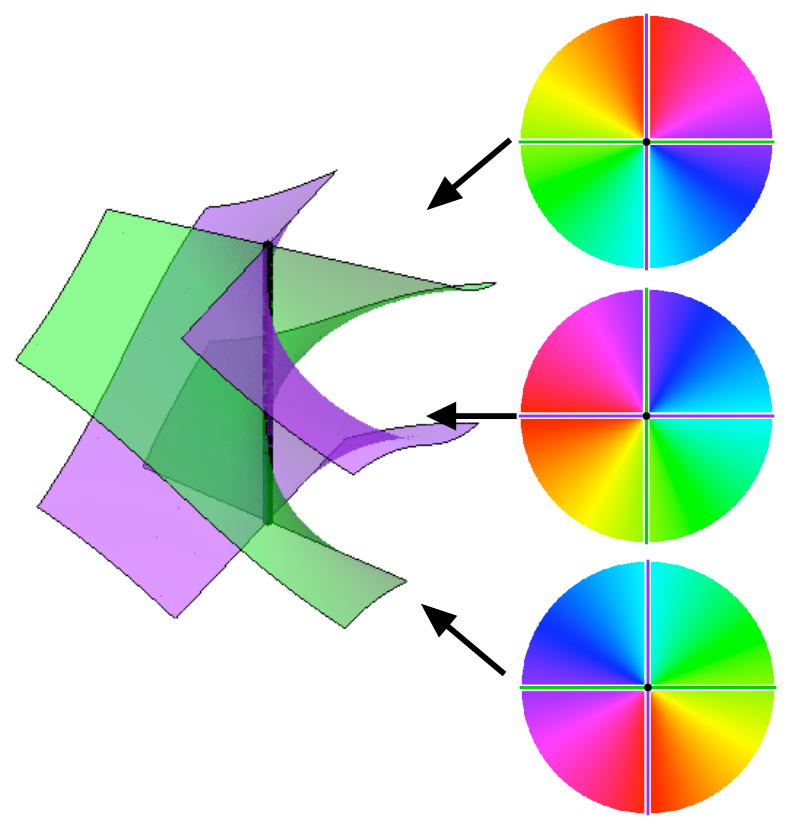

Fig. 4 The helical zero contour surfaces of the screw-dislocated plane wave (3), for $k>0$. In this example, transverse phase distribution rotates uniformly along the dislocation line in a left-handed sense.

dislocated plane wave [1, 3],

$$
\psi_{\text {screw }}=(x+\mathrm{i} y) \exp (\mathrm{i} k z),
$$

which satisfies the three-dimensional wave equation and whose phase surfaces are helicoids intersecting on the $z$-axis (Fig. 4), although the wave diverges unphysically away from the $z$-axis. The rate of rotation in this example is uniform, although in general the rotation is nonuniform and is related to the vortex core anisotropy [8]. Any small perturbation of this field moves the intersection of the helices, yielding a helical vortex [3]. It is straightforward to show $[8$ that the handedness of the helices is determined by the sign of the scalar product of $\boldsymbol{\Omega} \cdot k \hat{\boldsymbol{z}}$ : left-handed for positive, and right-handed for negative.

Intersecting real and imaginary contour surfaces assist in the understanding of points of vortex topology change with the variation of an additional parameter, namely loop nucleation or reconnection [4, 6, 16, 17. Near these events, $\psi$ can always undergo a local transformation $\psi \rightarrow \psi \exp (\mathrm{i} \alpha)$ making the $\eta=0$ surface locally flat (to second order) [16. The curvature of the transformed $\xi=0$ surface then determines the nature of the event: positive gaussian curvature gives a loop nucleation/annihilation, and negative gaussian curvature gives reconnection, the precise event taking place where the two surfaces have a common normal, i.e. $\boldsymbol{\Omega}=0$ (Figs. 5 and 6). These topology-changing events occur in several situations where an additional parameter (such as the amplitude of a component in the superposition) is changed [18, most simply in the superposition of four plane waves [19]. Further details of the mathematical structure of generic topology-changing events, and their involvement with lines of vanishing vorticity, are described in Ref. [16].

For the remainder of this article, I will concentrate on fields algebraically composed of the two following complex scalar functions

$$
\begin{aligned}
& u=r^{2}-1+2 \mathrm{i} z, \\
& v=2(x+\mathrm{i} y),
\end{aligned}
$$




\section{Topologica}

Vol. 2, 2009
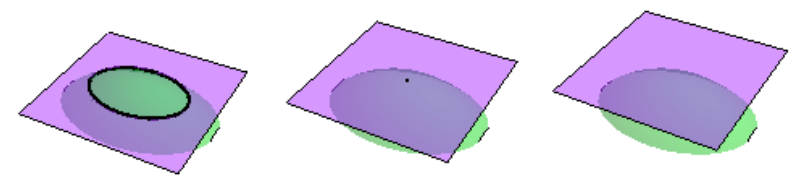

Fig. 5 Loop annihilation/nucleation, illustrated by the evolution of the real and imaginary zero surfaces with respect to an extra parameter. One of these surfaces is flat, the other has positive Gaussian curvature. The loop vanishes/appears at a point where the two surfaces touch (have a common normal direction).
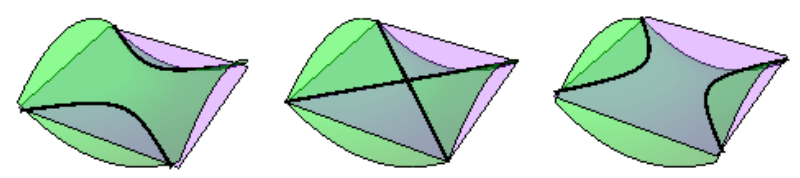

Fig. 6 Reconnection, illustrated by the evolution of the real and imaginary zero surfaces. One of these surfaces is flat, the other has negative Gaussian curvature (is saddle-shaped). The topological connections change at a point where the two surfaces touch (have a common normal direction).

with $r=|\boldsymbol{r}|=\sqrt{x^{2}+y^{2}+z^{2}}$. The vortex configurations of $v$ and its powers have already been discussed. The zero contour surfaces of the function $u$ consist of a sphere of radius unity centred on the origin, and the plane $z=0$. These clearly intersect on a circle of unit radius in the $x y$-plane (Fig. 7). A feature of this particular field is that its real part has a zero surface which is compact (i.e. closed and of finite area); embedding in a plane wave, the field $u \exp (\mathrm{i} z)$ has an additional disk of wavefront terminating on the vortex loop. This contrasts with wave dislocation loops usually encountered in solutions of the paraxial or Helmholtz equations, which

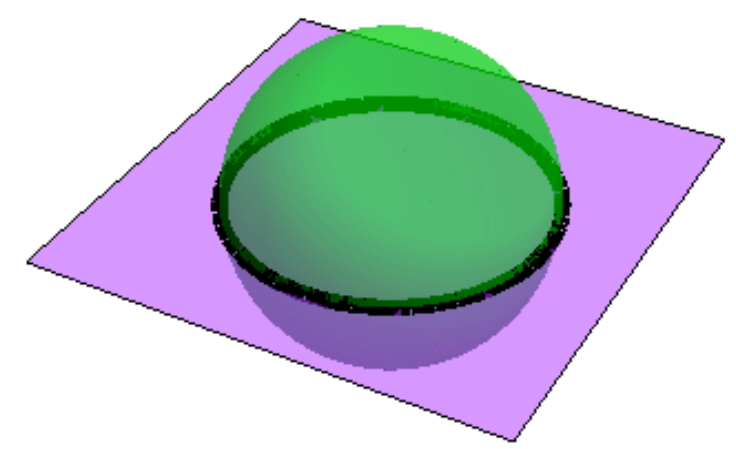

Fig. 7 The zero contour surfaces, sphere and plane, for the function $u$ of $(4)$, intersecting on a circle. 


\section{a}

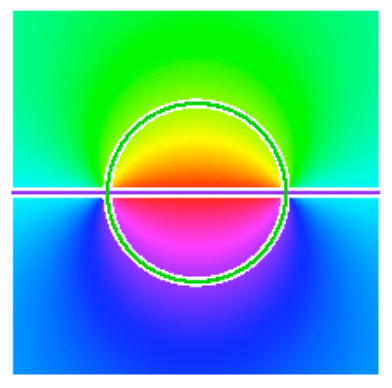

b

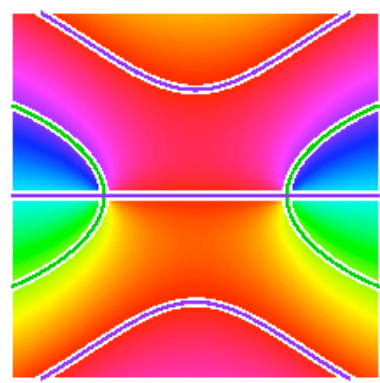

Fig. 8 Phase plots in the $x z$-plane of the fields (a) $u$; (b) $\psi_{\text {edge }}$, with the real (green) and imaginary (blue) zero contours. The green loop forms a closed phase surface in (a), but is a punctured sheet in (b).

usually bound 'punctured wavefronts' rather than an extra pieces of wavefront 3 , 20]. This is the case for the well-known closed wave edge dislocation, solving the Helmholtz equation [1, 3]

$$
\psi_{\text {edge }}=\left(x^{2}+y^{2}-1+2 \mathrm{i} z / k\right) \exp (\mathrm{i} k z)
$$

The phase profiles in the $x z$-plane for the two circular vortex loop fields, $u \exp (\mathrm{i} z)$ and $\psi_{\text {edge }}$, are given in Fig. 8. It has been shown [20] that propagating waves with simple edge dislocation loops must be of the punctured wavefront type.

As above with Fig. 2, it is also possible to consider the zero surfaces of powers of $u$, such as $u^{2}$. The $\eta=0$ surface now consists of the sphere and plane surfaces of $u$, and the $\xi=0$ surface is the union of two spheres of radius $\sqrt{2}$ centred at $(0,0, \pm 1)$. Although such high-strength loops are possible in solutions of the Helmholtz equation, they are prohibited in the paraxial equation [5].

As mentioned above, products of fields have the vortices of their individual components. For instance, the product $u v$ has a circular transverse vortex loop threaded by an axial vortex line (Fig. 9). This figure illustrates an important topological result, the screw theorem [4, 5, 8, 21, 22, 23]: the quantized number of twists around a closed singularity loop (properly, the linking number of a phase ribbon local to the vortex) equals the vortex strength threading the loop. This follows from considering the necessary topological structure of continuous complex scalar field, and relates a quantity associated with vortex lines (i.e. linking number) with zero contour surface topology (i.e. surface genus) - it is impossible to have one without the other. This result is similar to the concept of helicity in the topology of vortices in fluid flow [24, 25, 26]. Because a single vortex threads the loop in Fig. 9, each of the $\xi, \eta$ surfaces in the vicinity of the loop (i.e. ribbons) contains a single twist, requiring the surfaces globally to have genus at least one (i.e. at least one hole, no simpler than a torus).

The two surfaces of Fig. 9 are in fact identical apart from a $90^{\circ}$ rotation about the $z$-axis, and each is an infinite surfaces of genus 1 (non-compact torus), topologically identical to the zero surfaces considered as wavefronts in Ref. 8]. Such fields are relatively easy to implement in superpositions of beams, as the modulus of the field $u v$ - and, more generally, $u^{m} v^{n}$ - is azimuthally symmetric and so can be found as a superposition of Laguerre-Gauss or Bessel beams of azimuthal order $m$ (as mentioned above, in paraxial beams, $m$ must be 1 ). The case $u^{2} v^{2}$ is shown in Fig. 10. Such a field was the basis of the knot and link construction of Ref. [5] in solutions of the Helmholtz equation - a slight perturbation of the field $u^{m} v^{n}$ unfolds the strength $n$ ring into $n$ filaments, which form a closed helix which undergoes $n / m$ 


\section{Topologica}

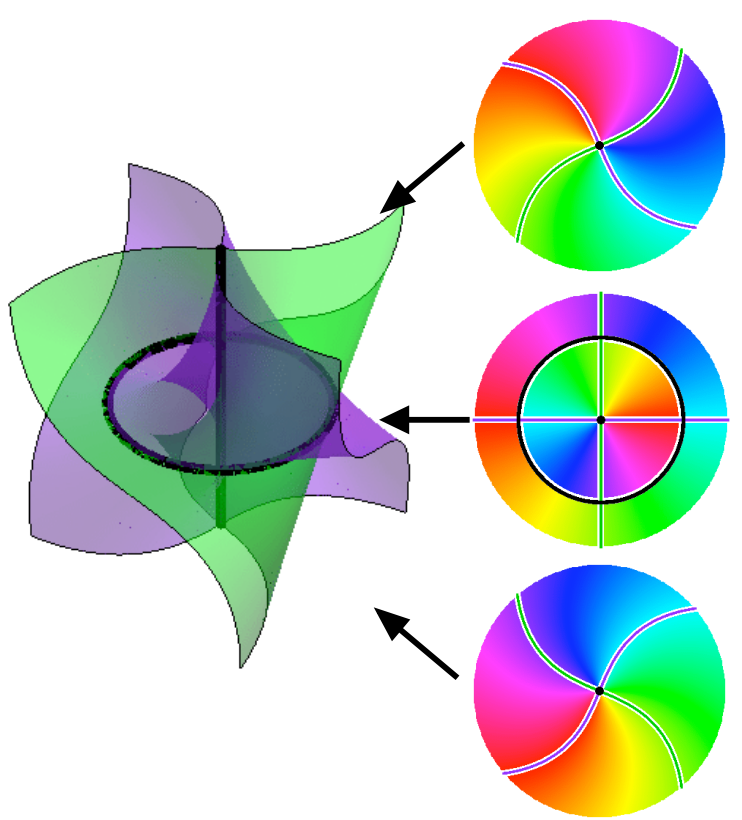

Fig. 9 The real and imaginary zero surfaces of the product field $u v$, intersecting on a closed loop and a straight line threading it. The two surfaces are the same up to a rotation by $90^{\circ}$, and are of genus 1 . Three transverse phase sections are shown; there is a planar vortex loop in the $z=0$ plane (which, as it is threaded by another vortex line, must topologically be a screw).

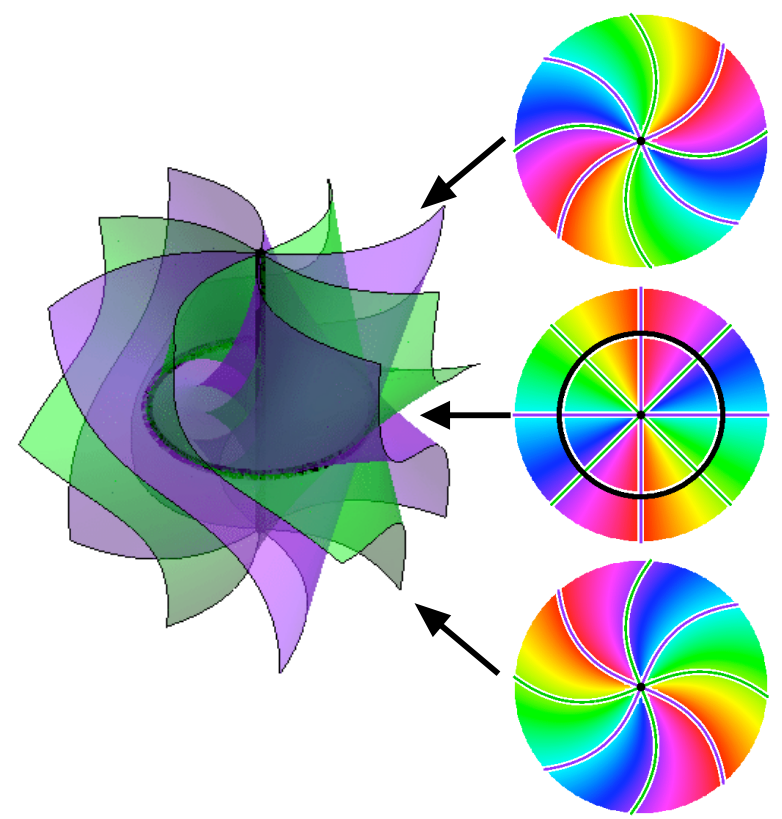

Fig. 10 The zero surfaces for the field $u^{2} v^{2}$, topologically corresponding to the unperturbed field configuration used to create the Hopf link in Ref. [5]. The closed loop in the $z=0$ has strength 2 , which cannot be physically realised in a paraxial beam [5]. 


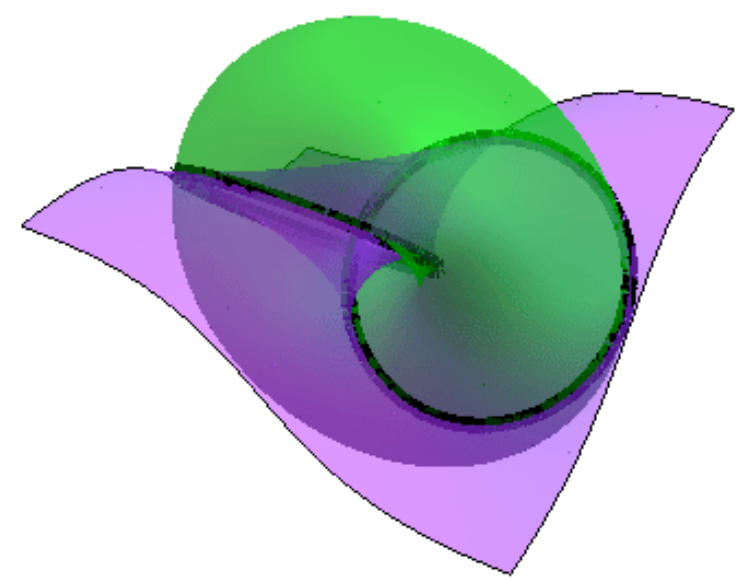

Fig. 11 The zero surface configuration of the field $U^{2}-V^{2}$, corresponding to a Hopf link (without additional vortex threading). The zero surfaces are each of genus 1 .

turns in one azimuthal circuit. This gives an $(m, n)$ torus knot, such as a Hopf link, for which $(m, n)=(2,2)$, and a trefoil knot, for which $(m, n)=(3,2)$. The knots and links formed by this construction are always threaded by $m$ vortices, unfolded from the strength $m$ axial vortex of the original unfolded configuration.

In fact, the fields $u$ and $v$ in (4) have a deeper mathematical significance. It turns out to be more natural to work with normalized forms

$$
\begin{aligned}
U & =\frac{r^{2}-1+2 \mathrm{i} z}{r^{2}+1}, \\
V & =\frac{2(x+\mathrm{i} y)}{r^{2}+1}
\end{aligned}
$$

Since these differ from $u$ and $v$ only by division by the positive real function $(1+$ $\left.r^{2}\right)^{-1}$, the zero contour surfaces discussed above are the same for $U$ and $V$. However, the pair $\{U, V\}$ has the additional property that it is normalized as a complex twodimensional vector, i.e. $|U|^{2}+|V|^{2}=1$. This implies that complex 2-vectors $\{U, V\}$ can be considered as points on the 3 -sphere (hypersphere) in four-dimensional space; the parameterization of $U$ and $V$ by $x, y, z$ means that (6) can be taken as the coordinates for stereographic projection of three-dimensional euclidean space to the 3 -sphere (i.e. point-set compactification of $\mathbb{R}^{3}$ ). Algebraic geometry [27] gives deep connections between polynomials in the two variables $U, V$ and knotted and linked phase singularities in the related complex scalar fields in three dimensions. Fields of this form occur in a range of nonlinear field theories, and are related to topological solitons [28, 29, 30]. The nonlinearities in the field equation stabilise the knot to some extent (so, for instance, topology-changing reconnections are avoided), and the knotted vortex configuration survives to the final numerical solution. This procedure has been successful in several different nonlinear physical theories, notably classical field theory [30, Bose-Einstein condensates [31, and reaction-diffusion equations 32].

A simple example of a topologically nontrivial field is $U^{2}-V^{2}$, whose singularities form the Hopf link (Fig. 11). This is straightforward to see since the field factorizes, $U^{2}-V^{2}=(U-V)(U+V)$, and each of these fields has a circular nodal ring, tilted at $90^{\circ}$ to each other, centred such that they link. The zero contours in this case are each of genus 1 , the $\xi=0$ surface being a closed torus, whereas $\eta=0$ is infinite (although is a compact torus in the 3 -sphere). However, there is nothing 


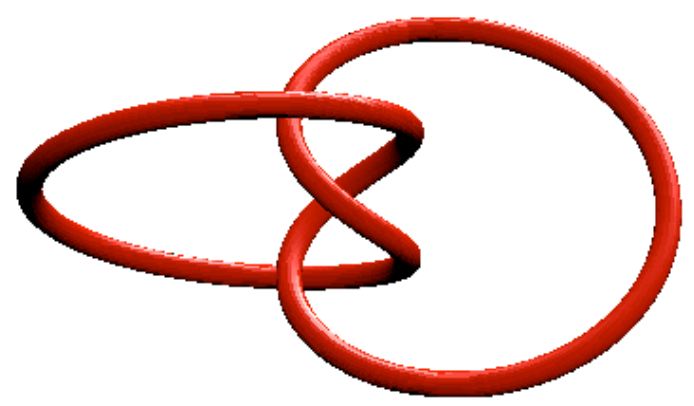

Fig. 12 The trefoil knotted nodal line of the complex scalar field $U^{3}-V^{2}$.

particularly special about this way of making a nodal link, as the product of any two fields with the correct nodal vortex rings will also contain a link.

Less trivial is the combination $U^{3}-V^{2}$, which gives a trefoil knot (Fig. 12). Unlike the other figures, the zero surfaces for this field are not plotted as the structure is too complicated. It is readily verified, however, that the $\xi=0$ surface is a twoholed torus (i.e. genus 3 compact surface), and $\eta=0$ is an infinite genus 3 surface. These two surfaces intersect to give the plotted trefoil knot; is difficult to see how to find such knotted nodal configurations without this sophisticated mathematics; unfortunately, it is not clear how to manipulate optical wave fields into a form similar to $U^{3}-V^{2}$, or the topologically equivalent $U^{2}-V^{3}$. The screw theorem implies that each knot has a self-linking number, related to the genus of its spanning Seifert surface and deducible from Călugăreanu's theorem [22, 23]. The threaded knots of [5, 6, 9, 10] affect this threading number, and the fibering of space by the surfaces of constant phase is different for a threaded knot (known to be possible in wave fields) and an unthreaded one (as yet undemonstrated in wave fields). Furthermore, more general fields $U^{m}-V^{n}$ correspond to unthreaded $(m, n)$ torus knots, and other forms of polynomial to other kinds of knot and link [27]; it is as yet unknown whether this approach can be used to generate unthreaded knotted and linked vortices in linear wave superpositions.

I have described some simple properties of phase singularity lines in threedimensional complex scalar fields as intersections of the zero contour surfaces of real and imaginary parts. Certain restrictions arise from the general case when the complex scalar field is restricted to satisfy a linear wave equation, particularly the Helmholtz and paraxial wave equations. Furthermore, the allowed phase singularity configurations differ in solutions of these two equations. To date there are very few explicit constructions of wave fields containing vortices with any measure of topological complexity, although it is possible that insight from other areas of vortex topology or topological field theories may provide insight into this problem.

\section{Acknowledgements}

I am grateful to Michael Berry, John Hannay, Robert King, John Nye, Kevin O'Holleran, Miles Padgett and Janne Ruostekoski for many discussions on this topic. My research is supported by the Royal Society of London.

\section{References}

[1] J. F. Nye and M. V. Berry, Proc. R. Soc. London, Ser. A 336, 165-90 (1974).

[2] M. V. Berry, in R. Balian, M. Kléman, and J.-P. Poirier, editors, Les Houches Session XXV - Physics of Defects, pp435-543 (North-Holland, 1981). 
[3] J. F. Nye, Natural focusing and fine structure of light: caustics and wave dislocations (Institute of Physics Publishing, 1999).

[4] M. R. Dennis, Ph.D. thesis, Bristol University, 2001.

[5] M. V. Berry and M. R. Dennis, Proc. R. Soc. London, Ser. A 457, 2251-63 (2001).

[6] M. V. Berry and M. R. Dennis, J. Phys. A: Math. Gen. 34, 8877-88 (2001).

[7] M. R. Dennis, New J. Phys. 5, 134 (2003).

[8] M. R. Dennis, J. Opt. A: Pure Appl. Opt. 6, S202-8 (2004).

[9] J. Leach, M. R. Dennis, J. Courtial and M. J. Padgett, Nature 432, 165 (2004).

[10] J. Leach, M. R. Dennis, J. Courtial and M. J. Padgett, New J. Phys. 7, 55 (2005).

[11] D. G. Grier, Nature 424, 810-6 (2003).

[12] I. Freund, Opt. Commun. 181, 19-33 (2001).

[13] Y. Y. Schechner and J. Shamir, J. Opt. Soc. Am. A 13, 967-73 (1996).

[14] M. V. Berry and M. R. Dennis, Proc. R. Soc. London, Ser. A 456, 2059-79 (2000).

[15] M. R. Dennis, in M. S. Soskin and M. V. Vasnetsov, editors, Singular Optics (Optical Vortices): Fundamentals and Applications, SPIE 4403 13-23 (2001).

[16] M. V. Berry and M. R. Dennis, J. Phys. A: Math. Theor. 40 65-74 (2007).

[17] J. F. Nye, J. Opt. A: Pure Appl. Opt. 6, S251-4 (2004).

[18] K. O'Holleran, M. R. Dennis and M. J. Padgett, JEOS-RP 1, 06008 (2006).

[19] K. O'Holleran, M. J. Padgett and M. R. Dennis, Opt. Express 14, 3039-44 (2006).

[20] J. F. Nye, J. V. Hajnal and J. H. Hannay, Proc. R. Soc. London, Ser. A 417, 7-20 (1988).

[21] A. T. Winfree and S. H. Strogatz, Physica D 9, 65-80 (1983).

[22] A. T. Winfree and S. H. Strogatz, Physica D 9, 333-45 (1983).

[23] A. T. Winfree, E. M. Winfree and H. Seifert, Physica D 17, 109-15 (1985).

[24] H. K. Moffatt, J. Fluid Mech. 35, 117-29 (1969).

[25] H. K. Moffatt and R. L. Ricca, Proc. R. Soc. London, Ser. A 439, 411-29 (1992).

[26] H. Aref and I. Zawadzki, Nature 354, 50-3 (1991).

[27] J. Milnor, Singular Points of Complex Hypersurfaces (Princeton University Press, 1968).

[28] N. Manton and P. Sutcliffe, Topological Solitons (Cambridge University Press, 2004).

[29] T. H. R. Skyrme, Proc. R. Soc. London, Ser. A 260, 127-38 (1961).

[30] L. Faddeev and A. J. Niemi, Nature 387, 58-61 (1997).

[31] J. Ruostekoski and J. R. Anglin, Phys. Rev. Lett. 86, 3934-7 (2001).

[32] P. M. Sutcliffe and A. T. Winfree, Phys. Rev. E 68, 016218 (2003). 